\title{
On Designing Truthful Spectrum Auctions for Variable Bandwidths
}

\author{
Tingting Chen ${ }^{1}$ Sheng Zhong ${ }^{2}$ \\ 1 Computer Science Dept., Oklahoma State University, tingting@cs.okstate.edu \\ 2 Computer Science and Engineering Dept., State University of New York at Buffalo, szhong@buffalo.edu
}

\begin{abstract}
Dynamic spectrum auctions have been considered a promising approach to effectively re-distribute spectrum resources in the secondary spectrum market. However, the existing spectrum auctions are limited to allocating spectrum in units of channels. Recently software defined radio technologies make exciting progress in operating radios with variable bandwidths. They push the need for designing more flexible spectrum auction frameworks that allow to allocate spectrum with variable bandwidth to the secondary user. In this paper, we design truthful spectrum auction frameworks in which secondary users can bid for, and then be actually allocated spectra with variable bandwidths. We first present a truthful framework for auctions of variable-bandwidth spectra in single collision domains, which can achieve system efficiency. Then, we propose a similar framework for multiple collision domains and rigorously show that it is also truthful. Results of extensive evaluations demonstrate that both of our spectrum auction frameworks for variable bandwidth are effective.
\end{abstract}

\section{INTRODUCTION}

With the fast-growing popularity of wireless network technologies, the wireless spectrum is becoming increasingly crowded. At the same time, measurements show that the spectrum allocated through static auctions is under utilized [20]. In order to utilize wireless spectrum more efficiently, recently a lot of attention has been given to building a secondary spectrum market, in which primary spectrum users can dynamically sell available channels to secondary users, through real-time auctions. Some spectrum auction frameworks have been proposed, with the goals of truthfulness, system efficiency, maximum revenue and/or fairness (e.g., [32], [14], [10]).

Dynamic spectrum auctions are effective in re-allocating spectrum resources and providing incentives for primary users to re-distribute their spectrum. However, we notice that the existing auction frameworks have the following limitation: In existing auction frameworks, available spectrum can only be auctioned in units of channels, i.e., each secondary user bids for one or multiple channels and the payments due are calculated based on the allocated channels. In contrast, recently advanced software defined radio technologies push the need for more flexible spectrum auction frameworks that allow to allocate spectra with variable bandwidths to secondary users. Specifically, these technologies enable the communication devices to operate with variable bandwidths, e.g., the 802.22 draft already includes the support for variable bandwidth [2] Moreover, recent studies (e.g., [31], [3]) have focused on building wireless networks in which the channel bandwidth can be adaptively changed. All the exciting progress in operating radios in variable bandwidths becomes an outcry for spectrum auction frameworks that allow primary users to allocate spectra in variable bandwidths, not just channels with a fixed bandwidth.

To the best of our knowledge, no existing work has studied variable-bandwidth spectrum auctions. In this paper, we aim to design a truthful auction framework that supports allocating variable bandwidths of spectra. Moreover, since system efficiency is a natural requirement when considering a spectrum auction, we also require our auction framework to be system efficient. Here system efficiency means that the system-wide total valuation should be maximized (see Section II for the precise definition).

The major challenges in designing such auction frameworks come from the nature of variable bandwidth. First, bidding for variable bandwidths makes it more complicated to describe a secondary user's demand for, and valuation of, the spectrum. Second, when allocating variable-bandwidth spectrum, we need to be more careful in order to avoid interference. Different from the channel allocation, where interference happens only when neighbors are assigned to exactly the same channel, in the variable-bandwidth spectrum allocation, interference exits as long as the overlap of neighbors' assigned spectra is not zero. Third, in order to guarantee truthfulness, it is more challenging to determine the amount of charge for a variable bandwidth spectrum than for a channel with a fixed bandwidth.

In addition to the above challenges, there is another factor that contributes to the hardness of the problem we try to solve: In this paper, we allow each secondary user to have multiple devices located at different positions. To bid for spectrum, each secondary user submits his valuation of spectrum for each device he owns. This further complicates the spectrum auction problem, because a secondary user can cheat in his claim of valuation on one device, which may benefit his other devices, so that he can obtain higher overall utilities. We will show how to tackle this difficulty in Section IV.

In order to support variable bandwidth spectrum auctions, we need to enable secondary users to submit their valuations for each possible bandwidth of spectrum on each device. So, unlike existing dynamic spectrum auction frameworks, our frameworks use a valuation function to describe each secondary user's valuation of all possible allocations of 
spectrum on each device. Hence, in an auction, each secondary user submits a set of valuation functions, not a set of numbers, to the primary user, as his bid. Valuation functions promise a more flexible form of bidding, by which variable bandwidth of spectrum can be auctioned.

Our main results in this paper are two spectrum auction frameworks for allocating variable bandwidths of spectra, in different settings. Our first framework, called VSA-S, is for Variable bandwidth $\underline{\text { Spectrum }}$ Auction in Single collision domains. We rigorously show that VSA-S is truthful. In addition, we show that VSA-S is system efficient. Our second framework, called VSA-M, is for Variable bandwidth Spectrum Auction in Multiple collision domains. We show that it is truthful just like VSA-S.

Our contributions can be summarized as follows:

- We are the first to study spectrum auctions for allocating variable bandwidth spectrum to secondary users.

- For variable bandwidth spectrum auction in single collision domains, we present an auction framework VSA-S. We rigorously show that VSA-S is truthful and system efficient.

- For variable bandwidth spectrum auction in multiple collision domains, we present a framework VSA-M that can be shown to be truthful.

- We have done extensive experiments to evaluate our spectrum auction frameworks and the results demonstrate that they have good performance.

The rest of this paper is organized as follows. In Section II, we present the technical preliminaries. In Sections III, we propose VSA-S and in Section IV we propose VSA-M. Section V is dedicated to evaluation results. Finally, we briefly review related literature in Section VI and then conclude in Section VII.

\section{TECHNiCAL PRELIMINARIES}

We consider spectrum auctions in which a primary user sells spectra with variable bandwidths to $K$ secondary users. Suppose that each secondary user $i$ has $D_{i}$ devices that need to use a bandwidth of spectrum. We use an interference graph to model the interference among the devices. If and only if two devices are within the interference range of each other, they become neighbors (i.e., connected by an edge) in the interference graph. (Throughout the paper, we use "neighbor" to refer to a neighbor in the interference graph.)

Assume that the information about available spectrum is stored in an occupancy database as required by FCC [1] and it changes slowly over time. ${ }^{1}$ Suppose that the frequency spectrum held by the primary user available for auction is $\left(f_{l}, f_{h}\right)$, where $f_{h}-f_{l}=W$. Unlike the previous works where secondary users submit numbers to represent their valuations in order to bid for channels, in our auctions each secondary user $i$ submits a valuation function set $V_{i}$ to the primary user. In particular, for each of his device

\footnotetext{
${ }^{1}$ The technologies of detecting primary users and accessing the spectrum over small timescales have been proposed by some existing works, e.g., [19].
}

$d, i$ submits a valuation function $v_{i, d}()$. The input of this valuation function is the bandwidth that is available to the device $(i, d)$. The output of this valuation function is the secondary user $i$ 's valuation on device $d$ of using this assigned bandwidth. Intuitively, $v_{i, d}()$ represents the $i$ 's satisfaction levels on device $d$ of using different bandwidths of spectra. We adopt the standard assumption from the literature of economics [17] that every valuation function $v_{i, d}()$ is strictly increasing and quasi-concave.

Upon receiving the valuation functions from the secondary users, the primary user first assigns spectrum with variable bandwidth to each winning device according to the valuation functions, and then computes the corresponding prices for using the spectra charged to the secondary users. Denote by $w_{i, d}$ the bandwidth of spectrum that is allocated to device $(i, d)$ in the auction.

\section{A. Truthfulness}

In the spectrum auction, the utility for each secondary user $i$ is decided by the profile of valuation function sets of all secondary users. We denote this profile by V. Formally, we have:

$$
\mathrm{u}_{i}(\mathrm{~V})=\sum_{1 \leq d \leq D_{i}} v_{i, d}\left(w_{i, d}\right)-p_{i}
$$

Intuitively, this means that the utility of secondary user $i$ is equal to $i$ 's total valuation of the spectra assigned to his devices minus the payment he needs to make for his use of spectra.

Given this game theoretic model, a spectrum auction in our scenario is truthful if and only if it is a dominant strategy equilibrium (DSE) [24] for all secondary users to submit their true valuation functions. Intuitively, a DSE guarantees that every player of the game has incentives to play the strategies specified by the DSE regardless of other players' behavior.

Definition 1. A spectrum auction is said to be truthful if it is a DSE for all secondary users to submit their true valuation function sets, i.e., for any secondary user $i$, assuming $V_{i}^{T}()$ is the true valuation function set of secondary user $i$, for any valuation function set $V_{i}^{A}()$ submitted by secondary user $i$, for any profile $V_{-i}$ of valuation functions submitted by all secondary users other than $i$,

$$
\mathbf{u}_{i}\left(V_{i}^{T}(), \mathrm{V}_{-i}\right) \geq \mathbf{u}_{i}\left(V_{i}^{A}(), \mathrm{V}_{-i}\right)
$$

Another goal of our design is to achieve system efficiency for the spectrum allocation. System efficiency is defined as follows.

Definition 2. A spectrum auction is system efficient if the spectrum allocation achieves the maximum system-wide total utility, i.e.,

$$
\left(w_{1,1}, \cdots, w_{i, d}, \cdots\right)=\arg \max _{w_{i, d}} \sum_{i} \sum_{d} v_{i, d}\left(w_{i, d}\right) .
$$




\section{AUCTION FrAMEWORK FOR SINGLE COLLISION DOMAIN}

In this section, we design and analyze a variable bandwidth spectrum auction framework, VSA-S, for the situation in which all devices are in a single collision domain. The design goal is to achieve the maximum system-wide total valuation and to guarantee truthfulness as well. Although the major contribution of this paper is the design of the spectrum auction framework for multiple-collision domains, thoroughly studying a simpler case allows us to better understand the nature of spectrum auction for variable bandwidth, and develop the key technique also used in multiple collision domains.

\section{A. Design of Auction Framework}

Now we first formalize the spectrum allocation problem in a single collision domain. Suppose that after the auction, each device $(i, d)$ has been assigned a bandwidth $w_{i, d}$ of spectrum. Since all devices are in a same collision domain, to avoid interference, we must have $\sum_{i} \sum_{d} w_{i, d}=W$. To maximize the system-wide total valuation, the spectrum allocation problem becomes the optimization problem

$$
\text { Maximize } \sum_{i} \sum_{d} v_{i, d}\left(w_{i, d}\right)
$$

Subject to: $\sum_{i} \sum_{d} w_{i, d}=W$, and $w_{i, d} \geq 0, \forall i, \forall d$.

One straightforward solution is to use the method of Lagrange multipliers [4] to find the maximum total valuation and the bandwidth of spectrum that each device should be allocated. In the payment step, for each winning device, the price of the bandwidth can be calculated in the fashion similar to the well-known VCG mechanism[27]. In particular, after temporarily removing the winning device $(i, d)$, the above spectrum allocation algorithm is executed again without $(i, d)$. Then the price for $(i, d)$ is calculated as the sum of the valuation increases of all other devices.

However, when the number of devices is large, to find the maximum total valuation and compute the payments will take a very long time, which is not desirable for realtime spectrum auctions. To remedy this problem, we design a method to enable allocating the spectrum with maximum total valuation with low computation cost and computing the payments for all devices in one round. In particular, we discretize the spectrum into slices. Assume that there is a constant $\epsilon$ such that all assigned bandwidths must be multiples of $\epsilon .^{2}$ Hence, we assign the bandwidth $W$ in slices of size $\epsilon$. We also discretize each submitted valuation function $v_{i, d}()$ to obtain a sequence of valuations: $b_{i, d, 1}$, $b_{i, d, 2}, \ldots, b_{i, d, W / \epsilon}$, where

$$
b_{i, d, j}=v_{i, d}(j \epsilon)-v_{i, d}((j-1) \epsilon) .
$$

Intuitively, each $b_{i, d, j}$ is a valuation of one slice of bandwidth of size $\epsilon$. To be more precise, $b_{i, d, j}$ is secondary user

\footnotetext{
${ }^{2}$ Since the precision of involved computing is limited, there must be such a small constant $\epsilon$.
}

$i$ 's valuation of the $j$ th such slice assigned to his device $d$. Note that, because $v_{i, d}()$ is strictly increasing and quasiconcave, we have

$$
0<b_{i, d, 1} \leq b_{i, d, 2} \leq \ldots \leq b_{i, d, W / \epsilon} .
$$

Suppose there are $N$ slices available to be assigned. Now the problem becomes a multi-unit auction of spectrum slices. We adopt the well-known multi-unit VCG auction mechanism to achieve system efficiency and truthfulness. The key ideas of multi-unit VCG are greedy spectrum allocation and opportunity-cost-based payment. In particular, we rank all valuations of spectrum slices (from all secondary users) and pick the $N$ largest. Our algorithm assigns the $N$ spectrum slices corresponding to $N$ highest slice valuations. A winning secondary user $i$ is charged the total valuation of all the newly assigned slices that replace the slices originally assigned to $i$ 's devices, when the system is without $i$.

Using the ideas above, we design VSA-S, the details of which are shown in Algorithm 1.

In VSA-S, $B^{\prime}(1)$ denotes the 1 st element of a sequence $B^{\prime}$. Similar notations are used throughout this paper. Note that when we write the details of VSA-S, each element of $B$ should not only be a valuation $b_{i, d, j}$; it should also contain the corresponding index $(i, d, j)$. Since the elements of $B^{\prime}, A, P, P_{i}$ all originate from $B$, they should similarly contain the indices.

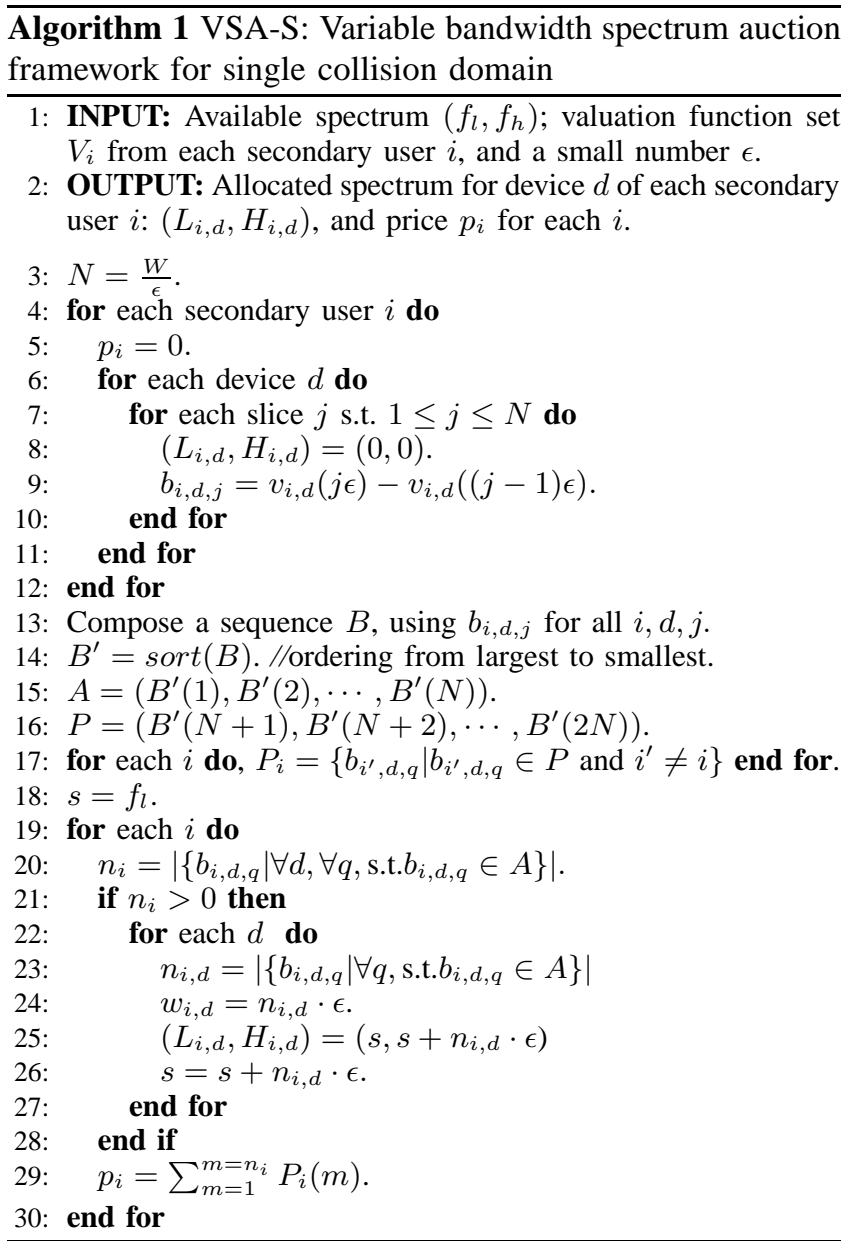




\section{B. Analysis of Framework}

Now we formally analyze SAS. We first prove the truthfulness of SAS. Then, we prove its optimality.

Theorem 3. In single collision domain, SAS is truthful.

Proof: Consider an arbitrary agent $i$. Given $V_{-i}$, the profile of valuation function sets submitted by all entities other than $i$, consider two possible strategies of agent $i$ : The first strategy is that agent $i$ submits its true valuation function set $V_{i}^{T}$, while the second is that agent $i$ submits an arbitrary valuation function set $V_{i}^{A}$. Clearly, these two strategies may lead to different values of variables in our SAS mechanism, and thus different payoffs of agent $i$. For convenience, we use superscript $T$ to denote the value of a variable when $V_{i}^{T}$ is submitted, e.g., $n_{i}^{T}$ is the value of $n_{i}$ when $V_{i}^{T}$ is submitted; correspondingly, we use superscript $A$ to denote the value of a variable when $V_{i}^{A}$ is submitted, e.g., $n_{i}^{A}$ is value of $n_{i}$ when $V_{e}^{A}$ is submitted. Those values that are not affected by agent $i$ 's submitted valuation function set remain without either superscript, e.g., $b_{i^{\prime}, d, j}$ for $i^{\prime} \neq i$. It is easy to get that

$$
\begin{aligned}
& \mathrm{u}_{i}\left(V_{i}^{T}, V_{-i}\right) \\
= & \sum_{d=1}^{D_{i}} v_{i, d}^{T}\left(w_{i, d}^{T}\right)-p_{i}^{T} \\
= & \sum_{d=1}^{D_{i}} v_{i, d}^{T}\left(n_{i, d}^{T} \cdot \epsilon\right)-\sum_{m=1}^{n_{i}^{T}} P_{i}^{T}(m) \\
= & \sum_{d=1}^{D_{i}} \sum_{m=1}^{n_{i, d}^{T}}\left(v_{i, d}^{T}(m \epsilon)-v_{i, d}^{T}((m-1) \epsilon)\right)-\sum_{m=1}^{n_{i}^{T}} P_{e}^{T}(m) \\
= & \sum_{d=1}^{D_{i}} \sum_{m=1}^{n_{i, d}^{T}} b_{i, d, m}^{T}-\sum_{m=1}^{n_{i}^{T}} P_{i}^{T}(m)
\end{aligned}
$$

Similarly,

$$
\begin{aligned}
& \mathrm{u}_{i}\left(V_{i}^{A}, V_{-i}\right)=\sum_{d=1}^{D_{i}} v_{i, d}^{T}\left(w_{i, d}^{A}\right)-p_{i}^{A}=\ldots \\
= & \sum_{d=1}^{D_{i}} \sum_{m=1}^{n_{i, d}^{A}} b_{i, d, m}^{T}-\sum_{m=1}^{n_{i}^{A}} P_{i}^{A}(m)
\end{aligned}
$$

Hence,

$$
\begin{aligned}
& \mathbf{u}_{i}\left(V_{i}^{T}, V_{-i}\right)-\mathbf{u}_{i}\left(V_{i}^{A}, V_{-i}\right) \\
= & \left(\sum_{d=1}^{D_{i}} \sum_{m=1}^{n_{i, d}^{T}} b_{i, d, m}^{T}-\sum_{d=1}^{D_{i}} \sum_{m=1}^{n_{i, d}^{A}} b_{i, d, m}^{T}\right) \\
& -\left(\sum_{m=1}^{n_{i}^{T}} P_{i}^{T}(m)-\sum_{m=1}^{n_{i}^{A}} P_{i}^{A}(m)\right) .
\end{aligned}
$$

Let ${B^{\prime}}_{i}^{T}$ (resp., ${B^{\prime}}_{i}^{A}$ ) be the subsequence of ${B^{\prime}}^{T}$ (resp., $B^{\prime A}$ ) that consists of all elements $b_{i, d, j}$ for all $d$ and all $j$. By our SAS mechanism, clearly we have that

$$
\sum_{d=1}^{D_{i}} \sum_{m=1}^{n_{i, d}^{T}} b_{i, d, m}^{T}=\sum_{m=1}^{n_{i}^{T}}{B^{\prime}}_{i}^{T}(m) .
$$

On the other hand, $\sum_{d=1}^{D_{i}} \sum_{m=1}^{n_{i, d}^{A}} b_{i, d, m}^{T}$ is the sum of $n_{i}^{A}$ elements of ${B^{\prime}}_{i}^{T}$. Let $M_{A}$ be the set of indices for these elements. Then, we have $\left|M_{A}\right|=n_{i}^{A}$ and that

$$
\sum_{d=1}^{D_{i}} \sum_{m=1}^{n_{i, d}^{A}} b_{i, d, m}^{T}=\sum_{m \in M_{A}}{B^{\prime}}_{i}^{T}(m) .
$$

Now let $B_{-i}^{\prime}$ be the sequence we obtain by removing all elements of $B^{\prime T}{ }_{i}$ from $B^{\prime T}$. Note that when we remove all elements of $B^{\prime}{ }_{i}^{A}$ from $B^{\prime A}$, we get the same sequence $B_{-i}^{\prime}$. It is not hard to see

$$
\begin{aligned}
& \sum_{m=1}^{n_{i}^{T}} P_{i}^{T}(m)=\sum_{m=N-n_{i}^{T}+1}^{N} B_{-i}^{\prime}(m) ; \\
& \sum_{m=1}^{n_{i}^{A}} P_{i}^{A}(m)=\sum_{m=N-n_{i}^{A}+1}^{N} B_{-i}^{\prime}(m) .
\end{aligned}
$$

Combining (2)(3)(4)(5), we get that

$$
\begin{aligned}
& \mathrm{u}_{i}\left(V_{i}^{T}, V_{-i}\right)-\mathrm{u}_{i}\left(V_{i}^{A}, V_{-i}\right) \\
= & \left(\sum_{m=1}^{n_{i}^{T}} B_{i}^{{ }^{T}}(m)-\sum_{m \in M_{A}}{B^{\prime}}_{i}^{T}(m)\right) \\
& -\left(\sum_{m=N-n_{i}^{T}+1}^{N} B_{-i}^{\prime}(m)-\sum_{m=N-n_{i}^{A}+1}^{N} B_{-i}^{\prime}(m)\right)
\end{aligned}
$$

We distinguish two cases:

Case A: $n_{i}^{T} \geq n_{i}^{A}$.

$$
\begin{aligned}
& \mathbf{u}_{i}\left(V_{i}^{T}, V_{-i}\right)-\mathbf{u}_{i}\left(V_{i}^{A}, V_{-i}\right) \\
= & \left(\sum_{m=1}^{n_{i}^{A}}{B^{\prime}}_{i}^{T}(m)-\sum_{m \in M_{A}}{B^{\prime}}_{i}^{T}(m)\right) \\
& +\sum_{m=n_{i}^{A}+1}^{n_{i}^{T}}{B^{\prime}}_{i}^{T}(m)-\sum_{m=N-n_{i}^{T}+1}^{N-n_{i}^{A}} B_{-i}^{\prime}(m) \\
\geq & 0+\left(n_{i}^{T}-n_{i}^{A}\right) B^{\prime T}{ }_{i}^{T}\left(n_{i}^{T}\right) \\
& -\left(n_{i}^{T}-n_{i}^{A}\right) B^{\prime}{ }_{i}\left(N-n_{i}^{T}+1\right) \\
= & \left(n_{i}^{T}-n_{i}^{A}\right)\left({B^{\prime}}_{i}^{T}\left(n_{i}^{T}\right)-B^{\prime}{ }_{-i}\left(N-n_{i}^{T}+1\right)\right)
\end{aligned}
$$

The inequality above is due to the fact that ${B^{\prime}}_{i}^{T}$ and ${B^{\prime}}_{-i}^{T}$ are both sorted from the largest to the smallest. From SAS, we can see that $B_{i}^{\prime T}$ has $n_{i}^{T}$ elements in $A^{T}$, i.e., in the top $N$ elements of $B^{\prime T}$. Hence,

$$
{B^{\prime}}_{i}^{T}\left(n_{i}^{T}\right) \geq B^{\prime T}(N) .
$$

This implies that $B^{\prime}{ }_{-i}$ has $N-n_{i}^{T}$ elements in the top $N$ elements of $B^{\prime T}$. Hence,

$$
B^{\prime}{ }_{-i}\left(N-n_{i}^{T}+1\right) \leq{B^{\prime}}^{T}(N) .
$$

Combining all the above three inequalities, we get that

$$
\mathrm{u}_{i}\left(V_{i}^{T}, V_{-i}\right)-\mathrm{u}_{i}\left(V_{i}^{A}, V_{-i}\right) \geq 0 .
$$

Case B: $n_{i}^{T}<n_{i}^{A}$. We partition $M_{A}$ into two subsets $M_{A, 1}\left(\left|M_{A, 1}\right|=n_{i}^{T}\right)$ and $M_{A, 2}\left(\left|M_{A, 2}\right|=n_{i}^{A}-n_{i}^{T}\right)$, such 
that the elements with indices in $M_{A, 1}$ are the largest $n_{i}^{T}$ elements with indices in $M_{A}$.

$$
\begin{aligned}
& \mathbf{u}_{i}\left(V_{i}^{T}, V_{-i}\right)-\mathbf{u}_{i}\left(V_{i}^{A}, V_{-i}\right) \\
= & \left(\sum_{m=1}^{n_{i}^{T}} B_{i}^{\prime T}(m)-\sum_{m \in M_{A, 1}}{B^{\prime}}_{i}^{T}(m)\right) \\
& -\sum_{m \in M_{A, 2}} B_{i}^{\prime T}(m)+\sum_{m=N-n_{i}^{A}+1}^{N-n_{i}^{T}} B_{-i}^{\prime}(m) \\
\geq & 0-\sum_{m \in M_{A, 2}} B_{i}^{\prime T}(m)+\left(n_{i}^{A}-n_{i}^{T}\right) B^{\prime}{ }_{-i}\left(N-n_{i}^{A}+1\right)
\end{aligned}
$$

Again, the inequality above is due to the fact that ${B^{\prime}}_{i}^{T}$ and $B^{\prime T}{ }_{-i}$ are both sorted from the largest to the smallest. Recall that ${B^{\prime}}_{i}^{T}$ has $n_{i}^{T}$ elements in the top $N$ elements of $B^{\prime T}$. Hence, $\left\{B_{i}^{\prime T}(m) \mid m \in M_{A}\right\}$ has at most $n_{i}^{T}$ elements in the top $N$ elements of $B^{\prime T}$. Consequently, $\left\{{B^{\prime}}_{i}^{T}(m) \mid m \in\right.$ $\left.M_{A, 2}\right\}$ has no element in the top $N$ elements of $B^{\prime T}$, which implies that, for all $m \in M_{A, 2}$,

$$
{B^{\prime}}_{i}^{T}(m) \leq{B^{\prime}}^{T}(N)
$$

On the other hand, similar to Case $\mathrm{A}, B^{\prime}{ }_{-i}$ has $N-n_{i}^{T}$ elements in the top $N$ elements of $B^{\prime T}$. Since $N-n_{i}^{A}+1<$ $N-n_{i}^{T}+1$,

$$
B^{\prime}{ }_{-i}\left(N-n_{i}^{A}+1\right) \geq{B^{\prime}}^{T}(N) .
$$

Combining all the above three inequalities, we get that

$$
\mathrm{u}_{i}\left(V_{i}^{T}, V_{-i}\right)-\mathrm{u}_{i}\left(V_{i}^{A}, V_{-i}\right) \geq 0 .
$$

To summarize, for both Case A and Case B we have shown that

$$
\mathrm{u}_{i}\left(V_{i}^{T}, V_{-i}\right) \geq \mathrm{u}_{i}\left(V_{i}^{A}, V_{-i}\right) .
$$

Hence, all entities submitting true valuation function sets is a DSE, which means SAS is truthful.

Theorem 4. (Optimality) In single collision domain, SAS achieves optimality.

Proof: So we need to show that it maximizes the total valuation.

Let $\Psi=\left\{w_{i, d} \mid \forall i, \forall d\right\}$ be the spectrum assignment result of SAS. Let $\Psi^{\prime}=\left\{w_{i, d}^{\prime} \mid \forall i, \forall d\right\}$ be the spectrum assignment result of an arbitrary different mechanism. It is easy to get that

$$
\begin{aligned}
& \sum_{i} v_{i}\left(w_{i}\right)-\sum_{i} v_{i}\left(w_{i}^{\prime}\right) \\
= & \sum_{i}\left(\sum_{\substack{1, \leq d \leq D_{i} \\
w_{i, d}^{\prime}=w_{i, d}}}\left(v_{i, d}\left(w_{i}\right)-v_{i, d}\left(w_{i, d}^{\prime}\right)\right)\right. \\
& +\sum_{\substack{1 \leq d \leq D_{i} \\
w_{i, d}^{\prime}<w_{i, d}}}\left(v_{i, d}\left(w_{i}\right)-v_{i, d}\left(w_{i, d}^{\prime}\right)\right) \\
& \left.+\sum_{\substack{1 \leq d \leq D_{i} \\
w_{i, d}^{\prime}>w_{i, d}}}\left(v_{i, d}\left(w_{i, d}\right)-v_{i, d}\left(w_{i, d}^{\prime}\right)\right)\right) \\
= & \sum_{i}\left(\sum_{\substack{1 \leq \leq d \leq D_{i} \\
w_{i, d}^{\prime}<w_{i, d}}}\left(v_{i, d}\left(n_{i, d} \cdot \epsilon\right)-v_{i, d}\left(n_{i, d}^{\prime} \cdot \epsilon\right)\right)\right) \\
& +\sum_{\substack{1 \leq d \leq D_{i} \\
w_{i, d}^{\prime}>w_{i, d}}}\left(v_{i, d}\left(n_{i, d} \cdot \epsilon\right)-v_{i, d}\left(n_{i, d}^{\prime} \cdot \epsilon\right)\right) \\
= & \sum_{i}\left(\sum_{\substack{1 \leq d \leq D_{i} \\
n_{i, d}^{\prime}<n_{i, d}}} \sum_{\substack{1, n_{i, d}^{\prime}+1 \\
n_{i, d}}} b_{i, d, m}-\sum_{\substack{1 \leq d \leq D_{i} \\
n_{i, d}^{\prime}>n_{i, d}}} m=\sum_{i, d}^{n_{i, d}^{\prime}} b_{i, d, m}\right)
\end{aligned}
$$

It is also clear that,

$\sum_{i} \sum_{\substack{1 \leq d \leq D_{i} \\ n_{i, d}^{\prime}<n_{i, d}}} \sum_{m=n_{i, d}^{\prime}+1}^{n_{i, d}} B^{\prime}(N)=\sum_{i} \sum_{\substack{1 \leq d \leq D_{i} \\ n_{i, d}^{\prime}>n_{i, d}}} \sum_{m=n_{i, d}+1}^{n_{i, d}^{\prime}} B^{\prime}(N)$

From SAS, we can see that

$\sum_{i} \sum_{\substack{1 \leq d \leq D_{i} \\ n_{i, d}^{\prime}<n_{i, d}}} \sum_{m=n_{i, d}^{\prime}+1}^{n_{i, d}} b_{i, d, m} \geq \sum_{i} \sum_{\substack{1 \leq d \leq D_{i} \\ n_{i, d}^{\prime}<n_{i, d}}} \sum_{m=n_{i, d}^{\prime}+1}^{n_{i, d}} B^{\prime}(N)$,

and that

$\sum_{i} \sum_{\substack{1 \leq d \leq D_{i} \\ n_{i, d}^{\prime}>n_{i, d}}} \sum_{m=n_{i, d}+1}^{n_{i, d}^{\prime}} b_{i, d, m} \leq \sum_{i} \sum_{\substack{1 \leq d \leq D_{i} \\ n_{i, d}^{\prime}>n_{i, d}}} \sum_{m=n_{i, d}+1}^{n_{i, d}^{\prime}} B^{\prime}(N)$.

From equations (6) (7) and (8), we can obtain that

$$
\begin{aligned}
& \sum_{i} \sum_{d=1}^{D_{i}} v_{i, d}\left(w_{i, d}\right)-\sum_{i} \sum_{d=1}^{D_{i}} v_{i, d}\left(w_{i, d}^{\prime}\right) \\
= & \sum_{i}\left(\sum_{\substack{1 \leq d \leq D_{i} \\
n_{i, d}^{\prime}<n_{i, d}}} \sum_{m=n_{i, d}^{\prime}+1}^{n_{i, d}} b_{i, d, m}\right. \\
- & \left.\sum_{\substack{1 \leq d \leq D_{i} \\
n_{i, d}^{\prime}>n_{i, d}}} \sum_{m=n_{i, d}+1}^{n_{i, d}^{\prime}} b_{i, d, m}\right) \geq 0 .
\end{aligned}
$$

Therefore, SAS achieves optimality.

\section{Auction Framework fOR Multiple Collision DOMAINS}

In the previous section, we have proposed a spectrum auction framework for single collision domain that guarantees truthfulness and system efficiency. However, if we consider multiple collision domains, the problem becomes very challenging. In particular, given the valuation function sets submitted by the secondary users, (even if they are 
all true), it is still very difficult to design a spectrum allocation algorithm that maximizes the total valuation in the system. Even one simplified version of our problem is already NP-Hard: In fact, when we simplify our problem to require that each device is allocated with a fixed bandwidth of spectrum, rather than variable bandwidth, the spectrum allocation problem in multiple collision domains becomes equivalent to the well-studied graph coloring problem[31]. Therefore, designing a system efficient spectrum allocation for variable bandwidths is NP-hard.

Given this fact of hardness, we have to weaken our objective for multiple collision domains. We will focus on designing a truthful and feasible spectrum auction framework, which is also challenging. In Section IV-D we will discuss in detail how to achieve approximate system efficiency with our proposed spectrum auction framework.

\section{A. Challenges in Design}

Our goal is to design a truthful spectrum auction framework that allocates spectra with variable bandwidths to the devices of secondary users without interference.

There are many challenges in designing such a spectrum auction framework. The first challenge is that we need to figure out how to charge the use of spectra with variable bandwidths. In most existing works (e.g., [32], [10], [14]) one key idea for ensuring truthfulness is to charge a winning secondary user a critical value of payment[22]. If a secondary user bids higher than his critical value, then he wins; If a secondary user bids lower than his critical value then he loses in the auction. For a secondary user, the existing works use a neighbor bidder's valuation on a channel as the critical value, which satisfies certain conditions. The neighbor is called critical neighbor or threshold neighbor[32], [10]. However, it is difficult to apply this idea directly to our problem, because for each allocated variable-bandwidth spectrum it is more complicated to find its corresponding critical value. To tackle this difficulty, we continue to adopt the idea used above of discretizing the spectrum into small intervals, to facilitate the spectrum allocation and price calculation.

Second, to avoid interference, most existing spectrum auction systems assign channels to secondary users sequentially, to make sure that the already assigned channels do not interfere with the allocation in the next steps. As a result, to compute the price for each winning secondary user, it needs to re-run the algorithm once. This is too expensive to be feasible for our scenario, since $\epsilon$ is supposed to be very small. In our design, instead of sequentially allocating spectra, we simultaneously allocate spectra to all devices and thus it only needs to run the algorithm once in total to compute the prices for all the winning secondary users. At the same time, we guarantee no interference in the system.

The third challenge comes from multiple devices that each secondary user may have. Suppose that a secondary user cheats in the valuation function on one of his devices. Even though this particular device does not obtain more spectrum by this cheating, it may still affect the spectrum allocation for the other devices owned by the cheating secondary user. The overall utility of the cheating secondary user, calculated as the sum of spectrum valuations from all his devices, may turn out higher eventually. This situation is difficult to deal with in order to guarantee truthfulness. We will show how we solve this problem later in this section.

\section{B. Design of Auction Framework}

Now we introduce our spectrum allocation algorithm, which consists of two steps. As the first step of our algorithm, to enable simultaneous allocation, we divide the spectrum $\left(f_{l}, f_{h}\right)$ into $\Delta$ intervals, and assign the center frequency of each device to one of the interval centers. We will discuss the range of parameter $\Delta$ and its relationship with system efficiency in Section IV-D. Neighbor devices in the interference graph are not assigned in the same intervals, and the devices belonging to the same secondary user are not assigned in adjacent spectrum intervals (in order to prevent secondary users from cheating by manipulating valuation of multiple adjacent devices). In our allocation algorithm, the spectrum of each device can grow from its center frequency to gradually farther spectrum slices, but not beyond the center frequency in the adjacent intervals. In this way, each device is competing for spectrum with the devices in the adjacent spectrum intervals. Consequently, its critical neighbor can be fixed by one run of the allocation algorithm.

As the second step of our algorithm, we assign spectrum slices to devices in a greedy fashion. In particular, VSA-M "grows" each device's spectrum following the rules below:

(1) No Overlaps. The growth of a device's spectrum must start from the spectrum slices closest to its center frequency and gradually go to farther spectrum slices, but not beyond the center frequency in the adjacent intervals. Once VSA$\mathrm{M}$ decides not to assign a symmetric pair to the device, the growth must terminate.

(2) Symmetric Allocation. The spectrum of a device grows in symmetric pairs of spectrum slices. Specifically, suppose that, in the first step, VSA-M has assigned the center frequency of device $(i, d)$ as $\mathrm{CF}_{\mathrm{i}, \mathrm{d}}$. Then, in the second step, after assigning symmetric pairs of spectrum slices to this device, the device's spectrum can only grow to $\left(\mathrm{CF}_{\mathrm{i}, \mathrm{d}}-n \epsilon, \mathrm{CF}_{\mathrm{i}, \mathrm{d}}+n \epsilon\right)$, where $n \geq 0$ is an integer.

The fundamental reason of using symmetric allocation is to guarantee truthfulness when secondary users have multiple devices. Suppose that we allow devices to grow spectrum in single slices. Without loss of generality, we assume that starting from the center frequency, the spectrum slice on the left side is always first considered to be assigned, and then the slice on the right side. A toy example scenario is shown in Fig. (1). Suppose that in the allocation algorithm, device $\left(i_{1}, d_{1}\right)$ is competing for slice $s_{1}$ with device $\left(i_{2}, d_{2}\right)$, and device $\left(i_{1}, d_{3}\right)$ is competing $s_{2}$ with device $\left(i_{2}, d_{2}\right)$, where $d_{1}$ and $d_{3}$ are from the same secondary user. If each secondary user bids truthfully, slice $s_{1}$ will be assigned to device $\left(i_{1}, d_{1}\right)$ and $s_{2}$ to $\left(i_{2}, d_{2}\right)$. Nevertheless, $i_{1}$ can submit a lower valuation on slice $s_{1}$, 


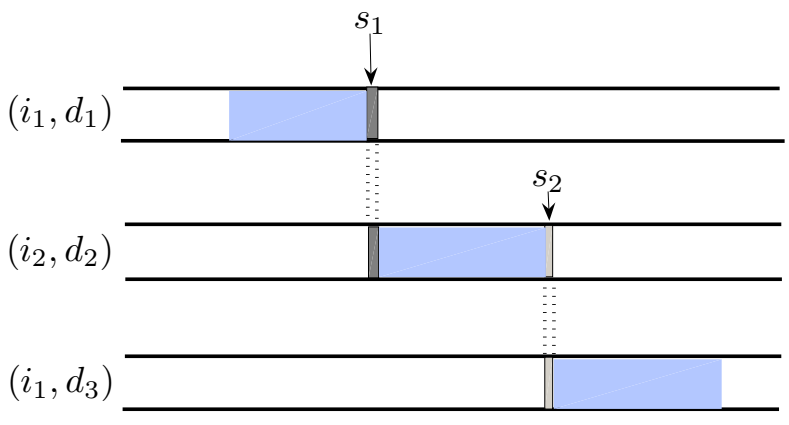

Already assigned spectrum

Fig. 1. Example for the necessity of symmetric allocation.

so that device $\left(i_{2}, d_{2}\right)$ will win $s_{1}$, and then the price for slice $s_{2}$ (valuation of $i_{2}$ on $s_{2}$ ) becomes lower for $i_{1}$. In this way, secondary user $i_{1}$ manipulates the price for $s_{2}$ by changing his valuation functions. Due to characterization of truthful auctions [22], we can see that this is not truthful. Therefore, we use the symmetric spectrum allocation, i.e., growth of spectrum in slice pairs, to prevent the secondary users from cheating.

(3) Greedy Assignment. A symmetric pair is assigned to a device only if the device's valuation of this pair of spectrum slices is the highest among all its neighbors' valuations of the conflicting pairs. Here a conflicting pair is a symmetric pair of spectrum slices for a neighbor (not for this device) that overlaps with this symmetric pair.

In Figure 2, we illustrate the rule (3) using an example. Here secondary user $i_{1}$ 's device $d_{1}$ and $i_{2}$ 's device $d_{2}$ are adjacent. For spectrum symmetric slice pair $P_{1}, P_{2}$ is the only conflicting pair. $i_{1}$ 's valuation on $P_{1}$ is $v_{e_{1}, d_{1}}(8 \epsilon)-v_{e_{1}, d_{1}}(6 \epsilon)$ and $i_{2}$ 's valuation on $P_{2}$ is $v_{e_{2}, d_{2}}(12 \epsilon)-v_{e_{2}, d_{2}}(10 \epsilon)$. If $v_{e_{1}, d_{1}}(8 \epsilon)-v_{e_{1}, d_{1}}(6 \epsilon)>$ $v_{e_{2}, d_{2}}(12 \epsilon)-v_{e_{2}, d_{2}}(10 \epsilon)$, then $P_{1}$ is assigned to $\left(v_{1}, d_{1}\right)$.

Once the growths of all devices' spectra are completed, VSA-M calculates the payment each secondary user needs to make for his use of spectra: This payment is equal to the sum of payments the secondary user needs to make for each symmetric pair of spectrum slices assigned to each of his devices. For each pair assigned to a device, the amount of payment due is determined by the device's neighbors belonging to other secondary users. We consider the valuations of conflicting pairs from such neighbors and use the highest such valuation as the payment.

In the example illustrated in Figure 2, assume that $d_{2}$ is the only neighbor of $d_{1}$ and that $e_{1} \neq e_{2}$. Then the payment due for usage of $P_{1}$ is the valuation of $P_{2}: v_{e_{2}, d_{2}}\left(B_{\ell}+\right.$ $12 \epsilon)-v_{e_{2}, d_{2}}\left(B_{\ell}+10 \epsilon\right)$.

The entire VSA-M framework is shown in Algorithm 2.

\section{Analysis of Auction Framework}

We have the following theorems regarding the truthfulness of VSA-M.

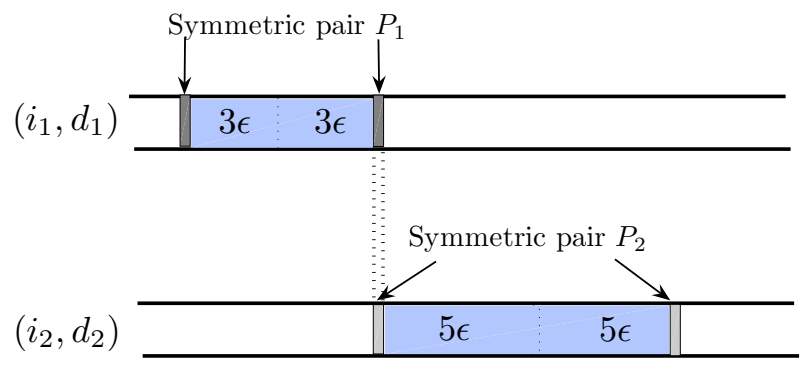

Already assigned spectrum

Fig. 2. Example for the 3rd restriction.

Theorem 5. In multiple collision domains, VSA-M is truthful.

Proof: Consider an arbitrary entity $i$. Given $V_{-i}$, the profile of valuation function sets submitted by all secondary users other than $i$, consider two possible strategies of $i$, i.e., submitting his true valuation function set $V_{i}^{T}$ and submitting an arbitrary valuation function set $V_{i}^{A}$. Notations such as $V_{i}^{T}, n_{i}^{T}, V_{i}^{A}$ and $n_{i}^{A}$ are defined similarly to in the proof of Theorem 3. (Recall that subscript $T$ means the value is for the scenario that $i$ submits $V_{i}^{T}$ and subscript $A$ means the value is for the scenario that $i$ submits $V_{i}^{A}$.) Those values that are not affected by $i$ 's submitted valuation function set remain without either superscript, e.g., $b_{i^{\prime}, d, j}$ for $i^{\prime} \neq i$.

We can easily get that

$$
\begin{aligned}
& \mathrm{u}_{i}\left(V_{i}^{T}, V_{-i}\right) \\
= & \sum_{d=1}^{D_{i}} v_{i, d}^{T}\left(w_{i, d}^{T}\right)-p_{i}^{T} \\
= & \sum_{d=1}^{D_{i}} v_{i, d}^{T}\left(n_{i, d}^{T} \cdot \epsilon\right)-\sum_{d=1}^{D_{i}} \sum_{m=1}^{n_{i, d}^{T}} p_{i, d, m}^{T} \\
= & \sum_{d=1}^{D_{i}} \sum_{m=1}^{n_{i, d}^{T}}\left(v_{i, d}^{T}(m \epsilon)-v_{i, d}^{T}((m-1) \epsilon)\right)-\sum_{d=1}^{D_{i}} \sum_{m=1}^{n_{i, d}^{T}} p_{i, d, m}^{T} \\
= & \sum_{d=1}^{D_{i}} \sum_{m=1}^{n_{i, d}^{T}} b_{i, d, m}^{T}-\sum_{d=1}^{D_{i}} \sum_{m=1}^{n_{i, d}^{T}} p_{i, d, m}^{T} .
\end{aligned}
$$

Similarly, we can obtain that

$$
\mathrm{u}_{i}\left(V_{i}^{A}, V_{-i}\right)=\sum_{d=1}^{D_{i}} \sum_{m=1}^{n_{i, d}^{A}} b_{i, d, m}^{T}-\sum_{d=1}^{D_{i}} \sum_{m=1}^{n_{i, d}^{A}} p_{i, d, m}^{A}
$$

Hence,

$$
\begin{aligned}
& \mathrm{u}_{i}\left(V_{i}^{T}, V_{-i}\right)-\mathrm{u}_{i}\left(V_{i}^{A}, V_{-i}\right) \\
= & \sum_{d=1}^{D_{i}}\left(\sum_{m=1}^{n_{i, d}^{T}} b_{i, d, m}^{T}-\sum_{m=1}^{n_{i, d}^{A}} b_{i, d, m}^{T}-\left(\sum_{m=1}^{n_{i, d}^{T}} p_{i, d, m}^{T}\right.\right. \\
& \left.\left.-\sum_{m=1}^{n_{i, d}^{A}} p_{i, d, m}^{A}\right)\right)
\end{aligned}
$$




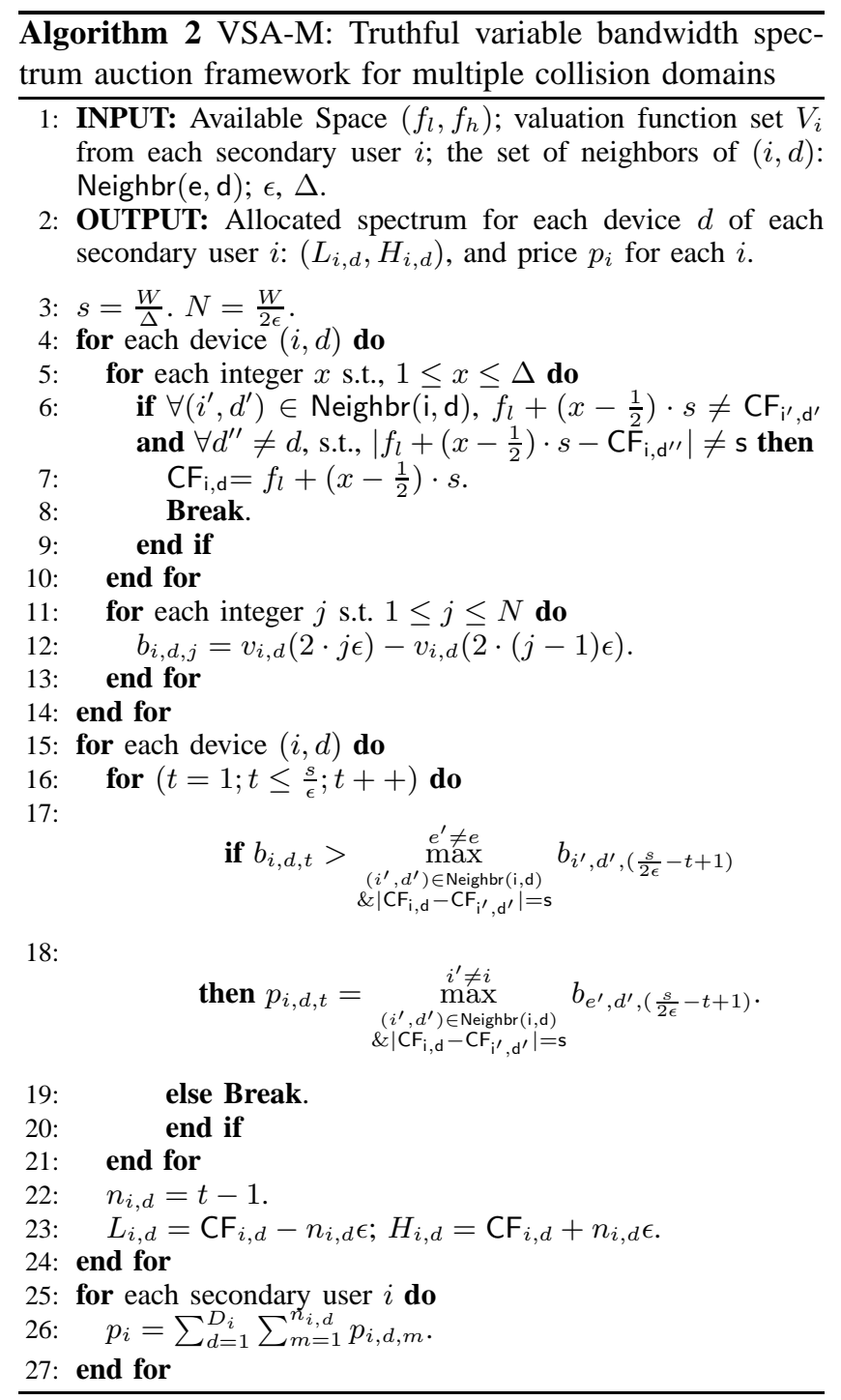

Our algorithm states that each

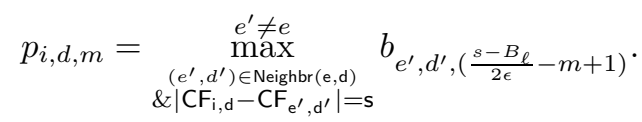

Since for each $m, \frac{s-B_{\ell}}{2 \epsilon}-m+1$ is a fixed number and not affected by $i$ 's submitted valuation function set, $p_{i, d, m}$ remains the same regardless of whether $i$ submits $V_{i}^{T}$ or $V_{i}^{A}$.

Then $\forall m$ s.t. $1 \leq m \leq \min \left(n_{i, d}^{T}, n_{i, d}^{A}\right)$ we have

$$
p_{i, d, m}^{T}=p_{i, d, m}^{A}
$$

. We distinguish two cases.

Case A. $n_{i, d}^{T} \geq n_{i, d}^{A}$.

$$
\begin{aligned}
& \mathrm{u}_{i}\left(V_{i}^{T}, V_{-i}\right)-\mathrm{u}_{i}\left(V_{i}^{A}, V_{-i}\right) \\
= & \sum_{d=1}^{D_{i}}\left(\sum_{m=n_{i, d}^{A}+1}^{n_{i, d}^{T}} b_{i, d, m}^{T}-\sum_{m=n_{i, d}^{A}+1}^{n_{i, d}^{T}} p_{i, d, m}^{T}\right) \\
= & \sum_{d=1}^{D_{i}}\left(\sum_{m=n_{i, d}^{A}+1}^{n_{i, d}^{T}}\left(b_{i, d, m}^{T}-p_{i, d, m}^{T}\right)\right) \\
\geq & 0
\end{aligned}
$$

The inequality above is due to the fact that, for any $m \leq$ $n_{i, d}^{T}$, we must have $b_{i, d, m}^{T} \geq p_{i, d, m}^{T}$.

Case B. $n_{i, d}^{T}<n_{i, d}^{A}$.

$$
\begin{aligned}
& \mathrm{u}_{i}\left(V_{i}^{T}, V_{-i}\right)-\mathrm{u}_{i}\left(V_{i}^{A}, V_{-i}\right) \\
= & \sum_{d=1}^{D_{i}}\left(-\sum_{m=n_{i, d}^{T}+1}^{n_{i, d}^{A}} b_{i, d, m}^{T}+\sum_{m=n_{i, d}^{T}+1}^{n_{i, d}^{A}} p_{i, d, m}^{A}\right) \\
= & \sum_{d=1}^{D_{i}} \sum_{m=n_{i, d}^{T}+1}^{n_{i, d}^{A}}\left(\max _{\substack{\left(e^{\prime}, d^{\prime}\right) \in \operatorname{Neighbr}_{(\mathrm{e}, \mathrm{d})} \\
\&\left|\mathrm{CF}_{\mathrm{i}, \mathrm{d}}-\mathrm{CF}_{\mathrm{e}^{\prime}, \mathrm{d}^{\prime}}\right|=\mathrm{s}}} b_{e^{\prime}, d^{\prime},\left(\frac{s-B_{\ell}}{2 \epsilon}-m+1\right)}\right. \\
\geq & \left.-b_{i, d, m}^{T}\right)
\end{aligned}
$$

This inequality holds because $\forall m$ s.t., $m>n_{i, d}^{T}$,

$$
b_{i, d, m}^{T} \leq \max _{\substack{\left(e^{\prime}, d^{\prime}\right) \in \operatorname{Neghbr}(\mathrm{e}, \mathrm{d}) \\ \&\left|\mathrm{CF} \mathrm{F}_{\mathrm{i}, \mathrm{d}}-\mathrm{CF}_{\mathrm{e}^{\prime}, \mathrm{d}^{\prime}}\right|=\mathrm{s}}} b_{e^{\prime}, d^{\prime},\left(\frac{s-B_{\ell}}{2 \epsilon}-m+1\right)}
$$

Therefore, it is a DSE for all secondary users to submit their true valuation function sets.

\section{System Efficiency in Multiple Collision Domains}

As we have mentioned, maximizing the total valuations in the system is NP-Hard. In our auction framework, the system efficiency depends heavily on the first step of the spectrum allocation, i.e., how to assign frequency centers to $\Delta$ different spectrum intervals. Intuitively, when $\Delta$ is smaller (i.e., each spectrum interval is larger), each device is more likely to obtain more spectrum in the allocation. However, $\Delta$ cannot be too small. In order to make sure that each devices can be assigned a center frequency without interference and that secondary users cannot benefit from cheating on the adjacent devices' valuations, $\Delta$ must satisfy the condition that $\Delta>\max \left(D_{\max }, 2 D_{\max }^{\prime}-1\right)$, where $D_{\max }$ is the maximum degree among all nodes in the interference graph and $D_{\max }^{\prime}$ is an highest number of a node's neighbors from the same secondary user. Since $D_{\max }+1$ is the lower bound of coloring index in a graph, it is easy to see that $\Delta>D_{\max }$ is a necessary condition to guarantee that each device is assigned a center frequency. Note that even if each device has been assigned a center frequency, some devices may still lose the auction and no bandwidth is allocated to them.

Given the condition on $\Delta$, we can extend the first step in our auction frame to increase the total valuation in the system. In particular, we can apply the approximate maximal independent set algorithm [25] to partition the devices into several independent sets, under the condition on $\Delta .{ }^{3}$ The devices in the same independent set are assigned to the same spectrum interval. In this way, an approximately maximum number of devices can share the same spectrum without interference, which increases the system efficiency.

\footnotetext{
${ }^{3}$ Note that the approximate maximal independent set algorithm needs to be applied once throughout the entire life of the system, as long as the interference graph does not change.
} 


\section{Evaluations}

We evaluate our spectrum auction frameworks in various settings. We carry out two sets of experiments for different objectives.

- The first set of experiments evaluates how the utility of a secondary user is affected by his possible cheating actions (in claiming his valuation function set). The results demonstrate that, when either VSA-S or VSA$M$ is used, secondary users' cheating actions never increase their own utilities.

- The second set of experiments evaluate the total valuation of allocated spectra in the system. The results demonstrate that, in a single collision domain when a cheating secondary user appears, VSA-S can prevent the total valuation of allocated spectra from decreasing; In multiple collision domains, VSA-M also achieves good system efficiency in spectrum utilization.

\section{A. Experiments Setup}

The experiments are performed using GloMoSim [18] on a laptop with $2.0 \mathrm{GHz}$ Centrino CPU and 1.96GB RAM. We modify GloMoSim to enable the use of variable spectra bandwidths, by setting the MAC layer parameters described in [5].

Unless specified otherwise, we assume that 3 secondary users, each of whom has 2 devices, are randomly located in an area of $300 \times 300 \mathrm{~m}^{2}$ (for single collision domain experiments), or $600 \times 600 \mathrm{~m}^{2}$ (for multiple collision domains experiments). The transmission power of each device is $16 \mathrm{dBm}$. The path loss is set to free space. In all experiments except those in Section V-C, we assume that the available band is $48 \mathrm{MHz}$ in DTV whitespace $(644 \mathrm{MHz}$ $692 \mathrm{MHz}$ ). All traffic is single hop UDP flows that are always backlogged. We set the packet size to 1500 Bytes.

In our experiments, we assume each valuation function is in one of the following two forms:

$$
\begin{gathered}
v_{i, d}\left(w_{i, d}\right)=\left\{\begin{array}{cl}
\beta_{i, d} \log \left(1+\gamma_{i, d} \cdot w_{i, d}\right) & \text { if } w_{i, d}<1 / \gamma_{i, d} \\
\beta_{i, d} \log 2 & \text { if } w_{i, d} \geq 1 / \gamma_{i, d} .
\end{array}\right. \\
v_{i, d}\left(w_{i, d}\right)=\left\{\begin{array}{cc}
\beta_{i, d} \sqrt{\gamma_{i, d} \cdot w_{i, d},} & \text { if } w_{i, d}<1 / \gamma_{i, d} \\
\beta_{i, d} \sqrt{2} & \text { if } w_{i, d} \geq 1 / \gamma_{i, d} .
\end{array}\right.
\end{gathered}
$$

The difference in devices' valuation functions is reflected in the difference in the values of $\beta_{i, d}$ and $\gamma_{i, d}$. When secondary users submit their valuation function sets, they may cheat by changing their values of $\beta_{i, d}$ and $\gamma_{i, d}$. When a secondary user is truthful, it should use the true values of $\beta_{i, d}$ and $\gamma_{i, d}$, denoted by $\beta_{i, d}^{\star}$ and $\gamma_{i, d}^{\star}$. We assume $\gamma_{i, d}^{\star}=1 /\left(n_{i, d} * 1 M\right)$.In experiments, we randomly set each $n_{i, d}$ as an integer in $[1,20]$. We set $\epsilon=1 \mathrm{MHz}$.

\section{B. Truthfulness and utilities}

In this set of experiments, we study the truthfulness of our spectrum auction frameworks. In particular, we evaluate how the cheating behavior of secondary users in bidding affects their own utilities. In each experiment, one random secondary user is picked to be the cheater; its claimed valuation function set has each $\beta_{i, d}$ (resp., $\gamma_{i, d}$ ) randomly chosen between 0 and $3 \beta_{i, d}^{\star}$ (resp., $\left.3 \gamma_{i, d}^{\star}\right) .{ }^{4}$ We measure the utility of the cheating secondary user in each experiment and also the same secondary user's utility when he behaves honestly. The difference is the secondary user's utility change brought by cheating. If the change is positive, then cheating brings higher utilities; otherwise, cheating does not benefit the secondary user.

Utilities in VSA-S We perform the above experiments on VSA-S, with 1000 runs using valuation functions in the form of (9) and another 1000 runs using valuation functions in the form of (10). We show the cumulative fraction plot of utility change in 3 (a). From Fig. 3 (a) we can observe that the utility change when cheating is never positive. In other words, secondary users never benefit from, and usually lose for, cheating. The average utility loss when cheating is 18.94. Similar observations can be made from Fig. 3 (b). In this case, the average utility loss when cheating is 14.62 . Overall, the truthfulness of VSA-S is verified.
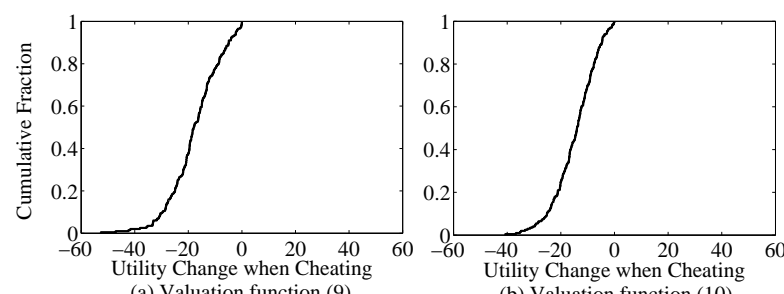

(a) Valuation function $(9)$

(b) Valuation function $(10)$

Fig. 3. Utility change for VSA-S. It shows that the utility change when cheating is never positive when VSA-S is used.

Utilities in VSA-M We also perform similar experiments on the auction framework for multiple collision domains. Fig. 4 shows the results for VSA-M. We can see that, if VSA-M is used, a secondary user's cheating behavior can never benefit himself (i.e., there is no positive utility change for cheating). Consequently, the truthfulness of VSA-M is verified.

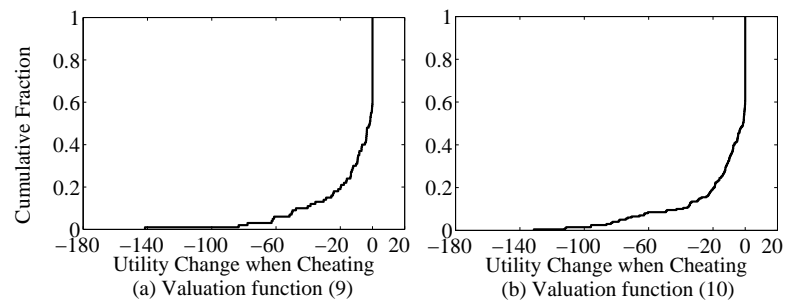

Fig. 4. Utility change for VSA-M. It shows that there is no positive utility change for cheating when VSA-M is used.

\footnotetext{
${ }^{4}$ We have this random choice of cheater and cheater's action because it is hard to predict who will be the cheater and how the cheater will behave in reality. By repeating this experiment for many times, we hope that at least some of the randomly picked cheating actions will be consistent with real cheaters' actions in reality.
} 


\section{Total Valuation}

The second set of experiments are to evaluate our two auction frameworks in terms of satisfying spectrum demands.

For the single collision domain, we measure the total valuation of allocated spectra for the case that all secondary users bid truthfully and compare it with the case that there is no payment scheme enforced in the system and one secondary user cheats in his submission of valuation functions. The result distributions shown in Fig. 5 demonstrate that VSA-S, which guarantees the truthfulness and system efficiency, can significantly increase ( $7.62 \%$ on average) the total valuation of allocated spectra with the presence of one cheating secondary user.

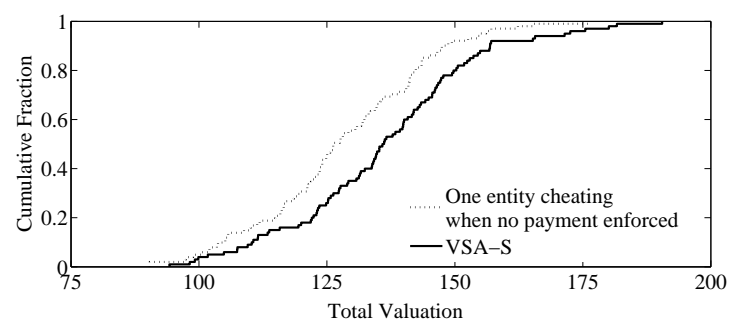

Fig. 5. Total valuation of allocated spectra in single collision domains.

For multiple collision domains, we measure the total valuations of allocated spectra for VSA-M in two different bands, the $2.4 \mathrm{GHz}$ ISM band and the DTV whitespaces, respectively. We assume that there are $80 \mathrm{MHz}$ available bandwidth in the $2.4 \mathrm{GHz}$ ISM band, and $48 \mathrm{MHz}$ available bandwidth $(644 \mathrm{MHz}-692 \mathrm{MHz})$ in DTV whitespaces. Fig. 6 shows the distributions of total valuation of allocated spectra of 100 runs, for our auction framework VSA-M, and a spectrum allocation algorithm that achieve approximate maximum total valuations using [25]. In the figure, we can see that, for both the $2.4 \mathrm{GHz}$ ISM band and the DTV whitespace, the total valuation of allocated spectra in the system remains at a high level, compared with the approximate algorithm. Since the there are more bandwidth available in $2.4 \mathrm{GHz}$ ISM band, system-wide total valuation is higher than that of DTV whitespace.
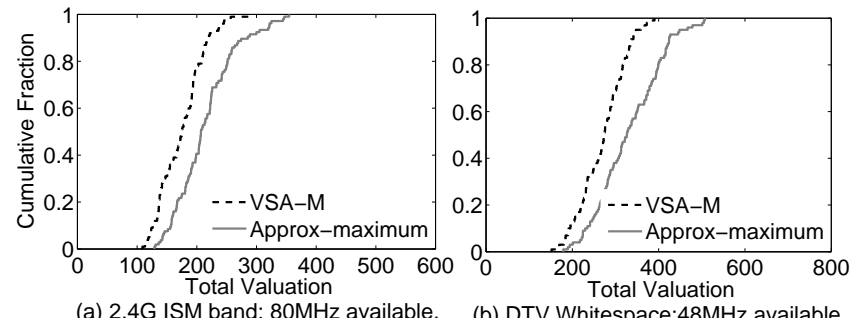

Fig. 6. Total valuation of allocated spectra in multiple collision domains.

\section{RELATED WORK}

Dynamic spectrum allocation with variable bandwidth has been studied extensively(e.g., [15], [31], [5], [11], [21],
[3]). In the KNOWS project [31], [30], [23], the concept of time-spectrum block is introduced and close-to-optimal central and distributed spectrum allocation algorithms [31] are proposed. In [21], Moscibroda et al. design algorithms to assign dynamic channel width that matches the traffic load. Their results show that load-aware dynamic spectrum allocation can significantly improve the spectrum utilization. Another important recent contribution [6] in DSA is on DTV whitespaces. In [6], in addition to providing some basic design rules and an architecture, Deb et al. also present a demand-based dynamic spectrum allocation algorithm that achieves high performance. These works on dynamical spectrum allocation consider the scenario that secondary users are not coordinated. The secondary users need to perform accurate and complicated spectrum sensing to avoid interfering with the primary user. In contrast, our work studies dynamic spectrum auctions, in which primary users can better control the usage of the available spectrum. Hence compared with these works above, we focus on a different system setting.

There is a considerable number of existing works on dynamic spectrum auctions (e.g., [13], [33], [32], [16], [14], [34], [10], [9]). Researchers produce nice and elegant spectrum auction frameworks with the goals of truthfulness, system efficiency, maximum revenue or fairness. For example, in [32], Zhou et al. propose a truthful and computationally efficient auction scheme; in [34], Zhou and Zheng make an important improvement by considering the incentives of the spectrum seller. Another truthful spectrum auction scheme is presented in [14] for generating more revenue from the auctions. As we have have mentioned, all these works on dynamic spectrum auctions only sell spectrum in units of channels. Our work provides more flexibility in selling unused spectrum with variable size of units.

There are also a number of works on non-cooperative channel assignment problem in wireless networks [12], [26], [7], [29], [8], [28]. For multiple radio devices, Felegyhazi et al. [7] introduce a strategic game model and obtain elegant theoretical results. After this work, Wu et al. [29] propose a solution based on strictly dominant strategies, and Gao et. al. [8] obtain interesting results in multi-hop networks. All these works are on assignment of fixed-width channels, rather than on allocation of spectra with variable bandwidths.

In a recent work [28], Wu et al. consider the noncooperative channel allocation problem, when the channel width is adaptive. They model the adaptive width channel allocation problem as a strategic game and design a payment scheme to guarantee the system converges to a dominant strategy equilibrium and achieve system optimality. Although this work makes good contributions to noncooperative adaptive-width channel allocation in general, our focus is in a different setting of secondary spectrum market and we allow more fine-grained spectrum allocation with the consideration of primary and secondary users' incentives. 


\section{CONCLUSION AND FutURE WORK}

Dynamic spectrum auctions are considered promising in utilizing the unused spectrum more efficiently in the secondary spectrum market. In this paper, we consider a more flexible form of dynamic spectrum auction, i.e., the spectrum can be sold in variable bandwidths. To solve this problem, we propose two spectrum auction frameworks with proved truthfulness and system efficiency properties, for single collision domain and multiple collision domains, respectively.

There are many possible ways to further improve our auction frameworks. For example, our auction framework for multiple collision domains can be further extended to achieve better approximation of system efficiency. Moreover, other design goals such as maximum revenue are also desirable for variable bandwidth spectrum auction. We leave these topics to future work.

\section{REFERENCES}

[1] F. 08-260. Second rep. and order and memorandum opinion and order. 2008

[2] I. 802.22. Ieee standards for wireless regional area network (wran) using white spaces. In http://www.ieee802.org/22/.

[3] P. Bahl, R. Chandra, T. Moscibroda, R. Murty, and M. Welsh. White space networking with wi-fi like connectivity. In Proc. of ACM SIGCOMM, 2009

[4] D. P. Bertsekas. Nonlinear Programming (Second ed.). Cambridge, MA.: Athena Scientific. ISBN 1-886529-00-0, 1999.

[5] R. Chandra, R. Mahajan, T. Moscibroda, R. Raghavendra, and P. Bahl. A case for adapting channel width in wireless networks. In Proc. of ACM SIGCOMM 2008, 2004.

[6] S. Deb, V. Srinivasan, and R. Maheshwari. Dynamic spectrum access in dtv whitespaces: Design rules, architecture and algorithms. In Proc. of ACM MOBICOM, 2009.

[7] M. Felegyhazi, M. Cagalj, S. S. Bidokhti, and J.-P. Hubaux. Noncooperative multi-radio channel allocation in wireless networks. In Proc. of IEEE INFOCOM, 2007.

[8] L. Gao and X. Wang. A game approach for multi-channel allocation in multi-hop wireless networks. In Proc. of ACM MOBIHOC, 2008.

[9] A. Gopinathan and Z. Li. A prior-free revenue maximizing auction for secondary spectrum access. In Proc. of IEEE INFOCOM, 2011

[10] A. Gopinathan, Z. Li, and C. Wu. Strategyproof auctions for balancing social welfare and fairness in secondary spectrum markets. In Proc. of IEEE INFOCOM, 2011.

[11] R. Gummadi and H. Balakrishnan. Wireless networks should spread spectrum based on demands. In Proc. of ACM HotNets-VII, 2008.

[12] M. M. Halldorsson, J. Y. Halpern, and L. E. L. etc. On spectrum sharing games. In Proc. of ACM PODC, 2004.

[13] J. Huang, R. Berry, and M. Honig. Auction-based spectrum sharing. Mobile Networks and Applications, 11(3), 2006.

[14] J. Jia, Q. Zhang, Q. Zhang, and M. Liu. Revenue generation for truthful spectrum auction in dynamic spectrum access. In Proc. of ACM MOBIHOC, 2009.

[15] T. Kamakaris, M. Buddhikot, and R. Iyer. A case for coordinated dynamic spectrum access in cellular networks. In Proc. of IEEE DySPAN, 2005.

[16] G. Kasbekar and S. Sarkar. Spectrum auction framework for access allocation in cognitive radio networks. In Proc. of ACM MOBIHOC, 2009.

[17] D. M. Kreps. A course in microeconomic theory. Princeton University Press, 1990.

[18] U. P. C. Laboratory. Information systems simulation library. http://pcl.cs.ucla.edu/projects/glomosim/.

[19] X. Liu, S. Huang, and Z. Ding. Opportunistic spectrum access in cognitive radio networks. In Proc. of IEEE INFOCOM, 2008

[20] M. McHenry, P. Tenhula, D. McCloskey, D. Roberson, and C. Hood. Chicago spectrum occupancy measurements and analysis and a longterm studies proposal. In In Proceedings of the first international workshop on Technology and policy for accessing spectrum, 2006.
[21] T. Moscibroda, R. Chandra, Y. Wu, S. Sengupta, P. Bahl, and Y. Yuan. Load-aware spectrum distribution in wireless lans. In Proc. of IEEE ICNP, 2008.

[22] R. Myerson. Optimal auction design. Mathematics of operations, 1981.

[23] S. Narlanka, R. Chandra, P. Bahl, and J. I. Ferrell. A hardware platform for utilizing the tv bands with a wi-fi radio. In Proc. of IEEE LANMAN.

[24] M. J. Osborne. A course in game theory. The MIT Press, 1994.

[25] M. T. S. Sakai and K. Yamazaki. A note on greedy algorithms for the maximum weighted independent set problem. Discrete Applied Mathematics, 2003

[26] R. W. Thomas, R. S. Komali, L. A. DaSilva, and A. B. MacKenzie. Joint power and channel minimization in topology control: A cognitive network approach. In Proc. of IEEE CogNet Workshop, 2007.

[27] W. Vickrey. Counterspeculation, auctions, and competitive sealed tenders. Journal of finance, 1961.

[28] F. Wu, N. Singh, N. Vaidya, and G. Chen. On adaptive-width channel allocation in non-cooperative, multi-radio wireless networks. In Proc. of IEEE INFOCOM, 2011.

[29] F. Wu, S. Zhong, and C. Qiao. Globally optimal channel assignment for non-cooperative wireless networks. In Proc. of IEEE INFOCOM, 2008.

[30] Y. Yuan, P. Bahl, R. Chandra, P. A. Chou, J. I. Ferrell, T. Moscibroda, S. Narlanka, and Y. Wu. Knows: Kognitiv networking over white spaces. In Proc. of IEEE DySPAN, 2007.

[31] Y. Yuan, P. Bahl, R. Chandra, T. Moscibroda, and Y. Wu. Allocating dynamic time-spectrum blocks in cognitive radio networks. In Proc. of ACM MobiHoc, 2007.

[32] X. Zhou, S. Gandhi, S. Suri, and H. Zheng. ebay in the sky: strategyproof wireless spectrum auctions. In Proc. of ACM MOBICOM, 2008.

[33] X. Zhou, S. Mettu, H. Zheng, and E. M. Belding. Traffic-driven dynamic spectrum auctions. In Proc. of IEEE Workshop SDR, 2008.

[34] X. Zhou and H. Zheng. Trust: A general framework for truthful double spectrum auctions. In Proc. of IEEE INFOCOM, 2009. 